\title{
INTERSECTING UNIONS OF CONVEX SETS IN $R^{n}$
}

\author{
MARILYN BREEN
}

ABSTRACT. Let $C=\left\{C_{a}\right.$ : $a$ in some index set $\left.I\right\}$ be a collection of convex sets, and let $\pi=\left\{C_{\alpha} \cup C_{\beta} a \neq \beta, C_{\alpha} C_{\beta}\right.$ in $\left.\mathcal{C}\right\}$. In this paper, various decomposition theorems are obtained for the set $\cap$ m.

1. Introduction. In [1], it is proved that if $\mathcal{C}$ is a collection of closed convex sets in the plane and if $\mathbb{M}=\{A \cup B: A, B$ distinct members of $\mathcal{C}\}$, then the set $\cap M$ is expressible as a union of three or fewer closed convex sets. In this paper, an attempt is made to obtain similar decompositions without the restriction that $\mathcal{C}$ be planar. Although several theorems are stated for an arbitrary linear topological space, restrictions on the convex sets reduce the setting to $R^{n}$, and all the theorems are essentially finite dimensional ones. Throughout the paper, aff $S$ and $\operatorname{ker} S$ will be used to denote the affine hull and kernel, respectively, for the set $S$. If $S$ is convex, $\operatorname{dim} S$ will denote the dimension of the affine hull of $S$, and for convenience, we say that the dimension of the null set is -1 .

2. Decomposition theorems for $\cap M$. The following easy lemmas will be useful.

Lemma 1. Let $\mathcal{C}=\left\{C_{\alpha}\right.$ : $\alpha$ in some index set $\left.I\right\}$ be a collection of sets, and let $\mathbb{M}=\left\{C_{a_{1}} \cup \ldots \cup C_{a_{k}}: a_{1}, \ldots, a_{k}\right.$ distinct member of $\left.I\right\}$. Then $x \in$ ก $\mathrm{M}$ if and only if there are at most $k-1$ members a in I for which $x \notin$ $C_{a^{*}}$

Lemma 2. Let $\mathcal{C}=\left\{C_{\alpha}: \alpha\right.$ in some index set $\left.I\right\}$ be a collection of convex sets in some linear topological space, and let $\mathbb{M}=\left\{C_{a_{1}} \cup \ldots \cup C_{a_{k}}\right.$ : $\alpha_{1}, \ldots, \alpha_{k}$ distinct members of $\left.I\right\}$. Then $\cap \mathcal{C} \subseteq \operatorname{ker}(\cap M)$.

Theorem 1. Let $\mathcal{C}=\left\{C_{a}\right.$ : a in some index set $\left.I\right\}$ be a collection of convex sets in some linear topological space, and assume that, for some $n \geq 1$, at least $n+1$ of these sets have dimension no greater than $n-1$.

Received by the editors April 1, 1974 and, in revised form, June 7, 1974.

AMS (MOS) subject classifications (1970). Primary 52A20, 52A40.

Key words and phrases. Unions of convex sets, maximal convex subsets. 
Letting $\mathbb{M}=\left\{C_{\alpha} \cup C_{\beta}: \alpha \neq \beta, C_{\alpha^{\prime}} C_{\beta}\right.$ in $\left.\mathcal{C}\right\}$, if $\operatorname{dim}$ aff $(\bigcap \mathbb{M})$ is at least $n$, then $\cap \mathbb{M}$ is a union of $n+1$ or fewer convex sets, each containing $\cap \mathcal{C}$. The number $n+1$ is best possible for every $n$.

Proof. We use an inductive argument. If $n=1$, then at least two members $A, B$ of $\mathcal{C}$ are singleton sets, $\cap \mathbb{M} \subseteq A \cup B$, and trivially $\cap \mathbb{M}$ consists of exactly two points.

Assume that the result is true for every integer $m, 1 \leq m \leq n-1$, to prove for $n$. There are two cases to consider.

Case 1. Suppose that there are $n+1$ affinely independent points $x_{1}, \ldots, x_{n+1}$ of $\bigcap \mathbb{M}_{\text {not }}$ in $\bigcap \mathcal{C}$. Then for each $i, 1 \leq i \leq n+1$, we may select a corresponding set $A_{i}$ in $\mathcal{C}$ with $x_{i} \notin A_{i}$. For any $C$ in $\mathcal{C}$ $\left\{A_{1}, \ldots, A_{n+1}\right\}, C$ necessarily contains each of the $n+1$ affinely independent points $x_{1}, \ldots, x_{n+1}$, and so $\operatorname{dim} C \geq n$. Hence the $A_{i}$ sets must be exactly those members of $\mathcal{C}$ which have dimension no greater than $n-1$, and the $A_{i}$ sets are necessarily distinct, $1 \leq i \leq n+1$. Then each $A_{i}$ must contain each of the $n$ points $x_{j}, j \neq i, 1 \leq j \leq n+1$. Since the points $x_{1}, \ldots$, $x_{n+1}$ are vertices of an $n$-dimensional simplex, each $A_{i}$ lies in the affine hull of a facet of the simplex. Therefore $A_{1} \cap \ldots \cap A_{n+1}=\varnothing$ and $\bigcap \mathbb{M}$ is just the union of the $n+1$ convex sets $B_{i}$, where $B_{i} \equiv \bigcap\{C: C$ in $\mathcal{C}, C \neq$ $\left.A_{i}\right\}=\left\{x_{i}\right\}, 1 \leq i \leq n+1$.

Case 2. If there are at most $k+1<n+1$ affinely independent points $x_{1}, \ldots, x_{k+1}$ of $\bigcap \mathbb{M}$ not in $\bigcap \mathcal{C}$, these points lie in a $k$-dimensional flat $\pi$ (and clearly we may assume $0 \leq k$ for otherwise the result is trivial). Select points $x_{k+2}, \ldots, x_{n+1}$ in $\cap$ so that $x_{1}, \ldots, x_{k+1}, x_{k+2}, \ldots, x_{n+1}$ are affinely independent. Then each of the $n-k$ points $x_{k+2}, \ldots, x_{n+1}$ must lie in $\cap \mathcal{C}$. For each of the members $A$ of $\mathcal{C}$ for which $\operatorname{dim} A \leq n-1$, there are no more than $n-(n-k)=k$ affinely independent points of $A$ in $\pi$, and $\operatorname{dim}(A \cap \pi) \leq k-1$. Hence $\mathcal{C}^{\prime} \equiv\{C \cap \pi: C$ in $\mathcal{C}\}$ is a collection of convex sets, at least $n+1>k+1$ of which have dimension no greater than $k-1$. Letting $\mathbb{M}^{\prime} \equiv\left\{C_{\alpha}^{\prime} \cup C_{\beta}^{\prime}: \alpha \neq \beta, C_{\alpha}^{\prime}, C_{\beta}^{\prime}\right.$ in $\left.\mathcal{C}^{\prime}\right\}$, dim aff $\left(\cap \pi^{\prime}\right)=$ $\operatorname{dim} \operatorname{aff}((\bigcap \pi) \cap \pi)=k$. Therefore, by our induction hypothesis, $\cap M^{\prime}$ is a union of $k+1$ or fewer convex sets, say $s_{1}^{\prime}, \ldots, s_{k+1}^{\prime}$, each containing ne'.

We assert that $\cap M$ is a union of the $k+1$ convex sets $S_{i} \equiv S_{i}^{\prime} \cup(\cap \mathcal{C})$, $1 \leq i \leq k+1$ : For $x$ in $\bigcap \mathbb{M}$ and $x$ not in any $S_{i}^{\prime}, 1 \leq i \leq k+1$, then $x \notin \pi$, so $x$ must belong to every $C$ in $\mathcal{C}$. Hence $S_{1} \cup \ldots \cup S_{k+1}=\bigcap \pi$. To show that each $S_{i}$ is convex, clearly we need only consider $r$ in $S_{i}^{\prime}, s$ in $\cap \mathcal{C}$ 
to show that $[s, r] \subseteq s_{i}$. Now by Lemma $2, s$ is in $\operatorname{ker}(\bigcap \mathcal{M})$, so $[s, r] \subseteq$ กM. If $s \in \pi$, the result is immediate since $\cap \mathcal{C}^{\prime} \subseteq S_{i}$. Otherwise, $[s, r)$ $\cap \pi=\varnothing$, so $[s, r) \subseteq \bigcap M \sim \pi \subseteq \cap \mathcal{C}$, and $[s, r] \subseteq\left(\bigcap^{\mathcal{C}}\right) \cup S_{i}^{\prime}=S_{i}$. Thus $s_{i}$ is convex, $1 \leq i \leq k+1$, and the assertion is proved, finishing Case 2 .

This completes the inductive argument, and we conclude that the statement of the theorem is true for every integer $n \geq 1$.

Remark. To see that the bound of $n+1$ in Theorem 1 is best possible, refer to Example 1 of this paper.

Theorem 2. Let $\mathcal{C}=\left\{C_{a}\right.$ : $a$ in some index set $\left.I\right\}$ be a collection of convex sets in $R^{n}, n \geq 1$, and let $\mathbb{M}=\left\{C_{\alpha} \cup C_{\beta}: \alpha \neq \beta, C_{\alpha}, C_{\beta}\right.$ in $\mathcal{C}_{\}}$. If there is an $n+1$ member subset $J$ of $I$ such that aff $\left(C_{\alpha} \cap(\cap M)\right) \neq \operatorname{aff}\left(C_{\beta} \cap(\cap M)\right)$ for $\alpha \neq \beta, \alpha$ in J, $\beta$ in 1 , then $\cap M$ is a union of $n+1$ or fewer convex sets, each containing $\cap \mathcal{C}$. The number $n+1$ is best possible.

Proof. The inductive argument of Theorem 1 may be suitably adapted to yield the result. The only significant difference appears in Case 2: As in Case 2, affinely independent points $x_{1}, \ldots, x_{k+1}, x_{k+2}, \ldots, x_{j+1}$ are. selected in $\cap M$ with $x_{1}, \ldots, x_{k+1}$ in the $k$-dimensional flat $\pi$ and not in $\bigcap \mathcal{C}$, and $x_{k+2}, \ldots, x_{j+1}$ in $\cap \mathcal{C} \sim \pi$, where $j=\operatorname{dim}$ aff $(\bigcap \Re)$ and $0 \leq k \leq j$. Then for $\alpha$ in $J, \beta$ in $I$, and $\alpha \neq \beta$,

$$
\operatorname{aff}\left(C_{a} \cap \pi \cap(\cap M t)\right) \neq \operatorname{aff}\left(C_{\beta} \cap \pi \cap\left(\cap \pi_{i}\right)\right) \text {, }
$$

for otherwise

$$
\begin{aligned}
\operatorname{aff}\left(\left[C_{\alpha} \cap \pi \cap\right.\right. & \left.(\cap M)] \cup\left\{x_{k+2}, \ldots, x_{j+1}\right\}\right) \\
& =\operatorname{aff}\left(\left[C_{\beta} \cap \pi \cap(\cap M)\right] \cup\left\{x_{k+2}, \ldots, x_{j+1}\right\}\right),
\end{aligned}
$$

and since $x_{k+2}, \ldots, x_{j+1}$ are in every $C$ in $\mathcal{C}$,

$$
\operatorname{aff}\left(C_{a} \cap(\cap M)\right)=\operatorname{aff}\left(C_{\beta} \cap(\cap M)\right),
$$

clearly impossible. Hence the induction hypothesis may be applied to the sets $\mathcal{C}^{\prime}$ and $\cap M^{\prime}$ of Case 2 to complete the argument.

The following example shows that the bound of $n+1$ in Theorems 1 and 2 is best possible.

Example 1. For $n \geq 1$, let $T$ denote an $n$-dimensional simplex and $\mathcal{C}$ the collection of facets of $T$. Then $\mathcal{C}$ has $n+1$ members, $\cap \mathcal{C}=\varnothing$, and $\cap M$ is the collection of points which lie in exactly $n$ facets of $T$. Hence 
กM is just the vertex set of $T$ and consists of $n+1$ isolated points.

Another kind of decomposition is given in Theorem 3.

Theorem 3. Let $\mathcal{C}=\left\{C_{a}: \alpha\right.$ in some index set $\left.I\right\}$ be a collection of closed convex sets, and let $\mathbb{M}=\left\{C_{a} \cup C_{\beta}: \alpha \neq \beta, C_{\alpha}, C_{\beta}\right.$ in $\left.C\right\}$. If for some $k \geq 1$ members $\alpha_{1}, \ldots, \alpha_{k}$ in $I, \operatorname{dim}\left(C_{a_{1}} \cap \ldots \cap C_{a_{k}}\right) \leq i,-1 \leq i \leq 2$, then ก) is a union of $k+i+1$ or fewer closed convex sets. The bound is best possible for every pair $k, i$.

Proof. For convenience of notation, let $C_{a_{i}}=C_{i}, 1 \leq i \leq k$, and define $D_{i} \equiv \bigcap\left\{: C\right.$ in $\left.\mathcal{C}, C \neq C_{i}\right\}$. For $x$ in $\cap M$, either $x$ lies in one of the closed convex sets $D_{i}, 1 \leq i \leq k$, or $x \in C_{1} \cap \ldots \cap C_{k}$.

We assert that the set $C_{1} \cap \ldots \cap C_{k} \cap(\cap M)$ is expressible as a union of $i+1$ or fewer closed convex sets: Define $C^{\prime} \equiv\left\{C_{1} \cap \ldots \cap C_{k} \cap C_{a}=\right.$ $C_{\alpha}^{\prime}: \alpha$ in $\left.I\right\}$, and let $\mathbb{M}^{\prime} \equiv\left\{C_{\alpha}^{\prime} \cup C_{\beta}^{\prime}: \alpha \neq \beta, C_{\alpha}^{\prime}, C_{\beta}^{\prime}\right.$ in $\left.\mathcal{C}^{\prime}\right\}$. Then $C_{1} \cap \ldots \cap$ $C_{k} \cap(\cap M)$ is exactly $\cap M^{\prime}$. If $i=2$, then $\mathcal{C}^{\prime}$ is a collection of closed convex sets in the plane, and by suitably adapting Theorem 1 in [1], $\cap M^{\prime}$ is a union of three or fewer closed convex sets, the desired result. In case $i=$ 1 , techniques used in [1] may be used to show that $\cap M^{\prime}$ is a union of 2 or fewer closed convex sets. For $i=0$ or $i=-1$, the result is trivial.

Therefore, $C_{1} \cap \ldots \cap C_{k} \cap(\cap M)$ is a union of $i+1$ closed convex sets, and hence $\cap M$ is a union of $k+i+1$ or fewer closed convex sets, finishing the proof of Theorem 3.

Example 2 reveals that the bound $k+i+1$ is best possible for every pair $k, i$.

Example 2. For a given $k \geq 1$ and for $-1 \leq i \leq 2$, if $k+i \geq 1$, let $\mathcal{C}$ denote the $k+i+1$ facets of a simplex $T$ in $\bar{R}^{k+\bar{i}}$. Then $k$ members of $\mathcal{C}$ intersect in an $i$-dimensional set, and $\bigcap M$, the vertex set of $T$, is a union of $k+i+1$ closed convex sets. If $k+i=0$, some member of $\mathcal{C}$ is empty, and $\cap M$ is convex.

Corollary. If $\mathcal{C}$ is a finite collection of closed convex sets in $R^{n}$ and $\operatorname{dim}(\cap \mathcal{C}) \leq 2$, then the corresponding set $\cap M$ is a union of $\sigma(n)+3$ or fewer closed convex sets, where $\sigma(n)=\max (n+1,2 n-4)$.

Proof. By a theorem of Katchalski [2], if all $\sigma(n)$ sets in $\mathcal{C}$ have at least a 3-dimensional intersection, then so does $\cap \mathcal{C}$. Hence if $\operatorname{dim}(\cap \mathcal{C}) \leq$ 2 , there are some $\sigma(n)$ sets in $\mathcal{C}$ whose intersection has dimension no more than 2. By Theorem $3, \cap M$ is a union of $\sigma(n)+2+1$ or fewer closed convex sets. 


\title{
REFERENCES
}

1. Marilyn Breen, Intersecting unions of maximal convex sets, Proc. Amer. Math. Soc. 39 (1973), 587-590. MR 47 \#7592.

2. Weir Katchalski, The dimension of intersections of convex sets, Israel J. Math. 10 (1971), 465-470. MR $46 \# 4367$.

DEP ARTMENT OF MATHEMATICS, UNIVERSITY OF OKLAHOMA, NORMAN, OKLAHOM A 73069

PROCEEDINGS OF THE

AMERICAN MATHEMATICAL SOCIETY

Volume 51, Number 2, September 1975

\section{A CHARACTERIZATION OF THE KERNEL OF A CLOSED SET}

\author{
MARILYN BREEN
}

ABSTRACT. Let $S$ be a closed subset of some linear topological space such that int ker $S \neq \varnothing$ and $\operatorname{ker} S \neq S$ o Let $\mathcal{C}$ denote the collection of all maximal convex subsets of $S$ and, for any fixed $k \geq 1$, let $\pi=\left\{A_{1} \cup \cdots \cup A_{k}: A_{1}, \ldots, A_{k}\right.$ distinct members of $\left.\mathcal{C}\right\}$. Then $\pi \neq \varnothing$ and $\cap \pi=\operatorname{ker} S$.

If $\mathcal{C}$ is the collection of all maximal convex subsets of some set $S$, it is easy to show that $\cap \mathcal{C}=$ ker $S$. This paper provides an interesting and perhaps surprising analogue of this well-known result. Throughout the paper, conv $S$, int $S$, and ker $S$ will be used to denote the convex hull, interior, and kernel, respectively, for the set $S$.

Further, we will make use of these familiar definitions: For points $x, y$ in a set $S$, we say $x$ sees $y$ via $S$ if and only if the corresponding segment $[x, y]$ lies in $S$. A subset $T$ of $S$ is said to be a visually independent subset of $S$ if and only if for every $x, y$ in $T, x \neq y, x$ does not see $y$ via $S$.

Received by the editors April 1, 1974 and, in revised form, June 7, 1974. AMS (MOS) subject classifications (1970). Primary 52A05.

Key words and phrases. Convex kernel, maximal convex subsets, unions of convex sets. 